\title{
Magnetic relaxation imaging with a fluxgate sensor scanner
}

\author{
Rühmer, D.; Heim, E.; Hirsch, A.; Piel, R.; Wawrzik, T.; Ludwig, F.; Schilling, M. \\ Institut für Elektrische Messtechnik und Grundlagen der Elektrotechnik \\ Technische Universität Braunschweig \\ Hans-Sommer-Straße 66 \\ D-38106 Braunschweig
}

\begin{abstract}
Fluxgate sensors are among the most sensitive low noise sensors for the magnetic flux density $\mathrm{B}$. We have developed a fluxgate scanner for the 2-dimensional magnetic relaxation imaging of magnetic nanoparticle distributions. In magnetorelaxometry (MRX), the magnetic moments of superparamagnetic nanoparticles are first aligned by an external magnetic field of the order of a few $\mathrm{mT}$, and after switching off the magnetic field the decay of the net magnetic signal is measured. In our setup, we employ a cylindrical wire-wound coil for the magnetization and racetrack fluxgates with a high permeability tape core for the detection. The fluxgate sensor with its electronics is mounted on a stand above the sample to be magnetically imaged. An x-y-table shifts the sample by maximum $200 \mathrm{~mm}$ in $\mathrm{x}$-direction and $180 \mathrm{~mm}$ in y-direction with a resolution of $100 \mu \mathrm{m}$ in both directions while typical scan steps are $1 \mathrm{~mm}$. The vertical distance of the sensor to the sample amounts to approx. $100 \mu \mathrm{m}$. In each point we measure the relaxation of magnetization of the sample to be tested repeatedly, and by displaying the resulting averaged change in magnetization we improve the signal-to-noise ratio. To increase the spatial resolution, data are deconvolved with a point-spread function, estimated from measurements on a pointshaped nanoparticle sample.
\end{abstract}

\section{Introduction}

Magnetorelaxometry (MRX) as described in [1] can be used to detect small amounts of magnetic nanoparticles which can be functionalized as specific marker. A major advantage of MRX compared to other techniques is its ability to distinguish between bound and unbound nanoparticles. This is important in biochemical assays since washing out unbound nanoparticles becomes redundant. In a MRX assay, the magnetic moments of superparamagnetic nanoparticles are first aligned by an external magnetic field of the order of a few $\mathrm{mT}$, and after abruptly switching off the magnetic field the decay of the net magnetic signal is measured.

In this paper, we describe a setup in which we combine our fluxgate MRX [1] with two-dimensional imaging to scan cell culture bags for immune cells. Inside these bags, magnetic nanoparticles can bind to the inner surface. This surface has been modified by plasma treatment to improve adherence of the immune cells to the inner walls enhancing cell division and thereby achieving fast growth rates of immune cells for therapy. Binding of magnetic nanoparticles only occurs where the plasma treatment has successfully created a surface with long term stability.

The important advantage is that the magnetic nanoparticles can test this surface modification within sterile environment also for longer periods of time: The normal growth period of the immune cells can amount to two weeks. Another major advantage of the MRX method is its ability to distinguish between nanoparticles that are bound to the inner surface and the unbound ones which freely move in the carrier liquid inside the bag. 


\section{Scanning setup}

The mechanical concept of our measurement setup (Fig. 1) is based on a commercially available two axis shifting table providing a maximum displacement of $20 \mathrm{~cm}$ in x-direction and $18 \mathrm{~cm}$ in y-direction. The displacement range has been selected to fit the size of standard $500 \mathrm{ml}$ cell culture bags. The sample to be tested is placed on top of a specifically designed holder ("sample table") which determines the zposition. For the measurement a flat surface of the sample is required. In order to achieve this with the flexible plastic bags we have designed a special cartridge system which holds the cell culture bags between two thin glass plates. The magnetic field sensor is located approximately $100 \mu \mathrm{m}$ above the cartridge. We make use of wire-wound fluxgate magnetometers which have been designed and manufactured at our institute [2]. The fluxgates consists of a permalloy racetrack-shaped core with a length of $40 \mathrm{~mm}$. Magnetization is done with two identical primary coils with a diameter of $1.2 \mathrm{~mm}$ and a length of $I_{\text {prim }}=37 \mathrm{~mm}$. The detection coil is $I_{s e c}=20 \mathrm{~mm}$ in length and positioned at the center of the core. We compensate the field using a separate compensation coil which is operated in a feedback loop. The magnetization field for the sample is generated by a cylindrical coil which surrounds the fluxgate and has a diameter of approximately $9 \mathrm{~cm}$ (Fig. 2).

(a)

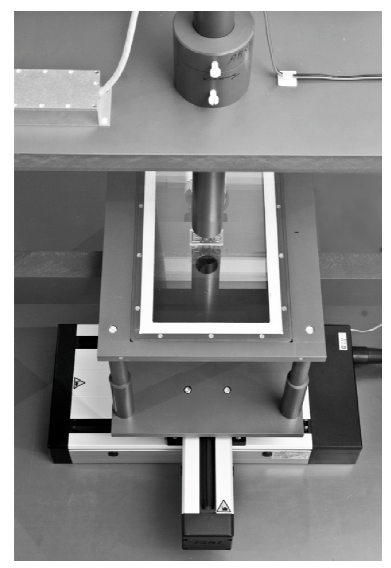

(b)

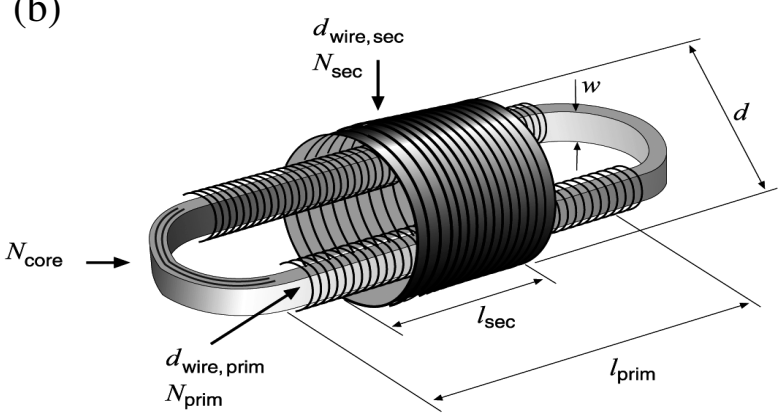

Figure 1: (a) Mechanical set-up of the MRX-scanner. (b) Schematic setup of wire-wound racetrack fluxgate

Since magnetorelaxometry requires fast switching of the magnetization field, the occurrence of spurious eddy currents needs to be taken into consideration. The first means to minimize this effect is the usage of non-metallic materials wherever possible. The only remaining metallic components are the off-the-shelf shifting table and the casing of the fluxgate signal processing circuit which needs to be shielded against RF. Hence, it is mandatory to maintain a minimum clearance between the sensor and the sample on the one side and the remaining metallic objects on the other side. A value of $25 \mathrm{~cm}$ has been found appropriate and this is the main drive for the overall dimensions of our scanning device of about $1 \mathrm{~m}$ in each direction.

Magnetizing the sample in order to align the magnetic moments of the nanoparticles is performed by applying a magnetic flux density at the surface of the sample of approximately $3 \mathrm{mT}$ for $1000 \mathrm{~ms}$. Using the described coil with a diameter of $9 \mathrm{~cm}$ a homogeneous magnetization of a large sample area is achieved and edge effects do not occur at the measurement point (Fig. 2). After switching off the magnetizing field the relaxation is measured for $750 \mathrm{~ms}$. Data are sampled with $100 \mathrm{kS} / \mathrm{s}$. As handling a two dimensional array of relaxation curves is not practical, we have developed a more visually oriented display format: A simple but yet effective method is to calculate the amplitude difference of two time-fixed points on the relaxation curve (Fig. 3). A gray scale value is assigned to this difference and is used to form a pixel on our graphic display. The gray scale is continuously adjusted to maximum and minimum amplitude differences. Pseudo colour display is also possible but less informative. We define the two points on the relaxation curve so that the comparatively fast relaxation of unbound particles is discarded and only the relatively slow relaxation process of the bound particles is considered. Alternatively we plan to apply curve fits, thus having alternative parameters for graphic display [3].

To improve signal-to-noise ratio, the relaxation is measured repeatedly up to 6-9 times followed by calculating the arithmetic mean. The time effort for scanning an exemplary square sample with a size of 5 


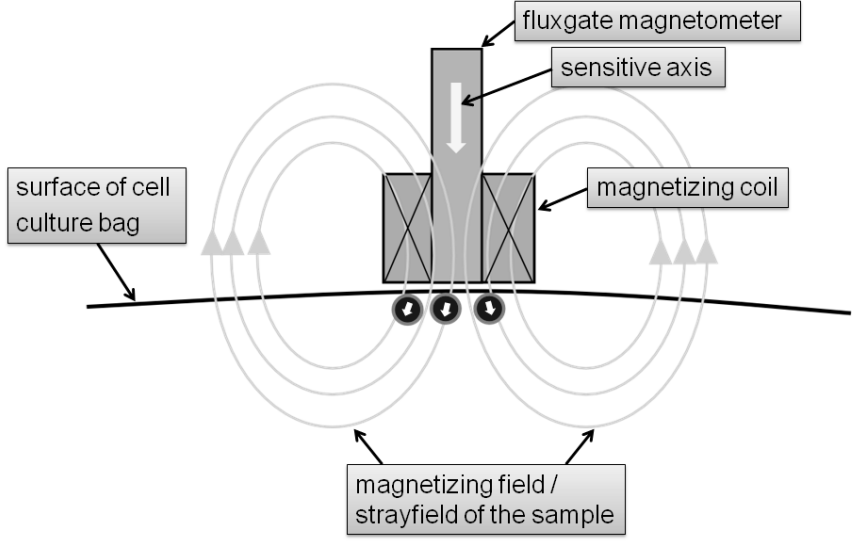

Figure 2: Schematic drawing of setup for magnetorelaxation imaging.

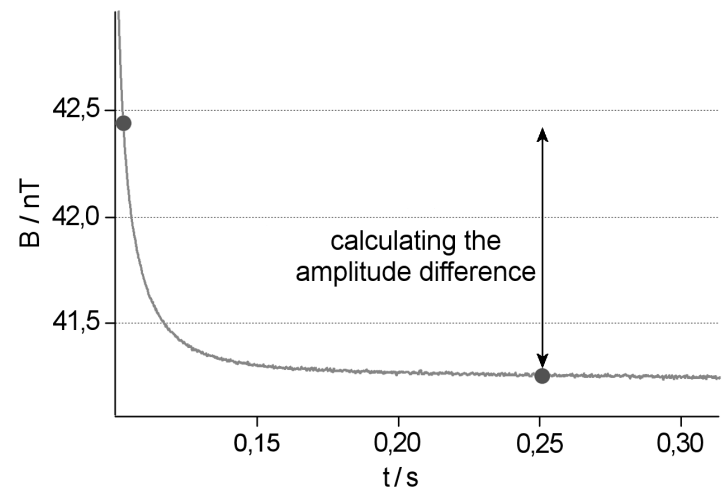

Figure 3: Estimation of signal from MRX curve for imaging.

$\mathrm{cm} \times 5 \mathrm{~cm}$ using scan steps of $1 \mathrm{~mm}$ and 9 averages is 11 hours. We plan to reduce the measurement time by using multiple uniform sensors forming an array and measuring several points at the same time.

\section{Scanning results}

The first two-dimensional scans of a $200 \mu \mathrm{l}$ sample of freeze-dried and thereby immobilized nanoparticles (which equates to $12 \mathrm{mg} \mathrm{Fe}$ or $1 \cdot 10^{14}$ nanoparticles) show rather blurred images. Although the sample has a diameter of $8 \mathrm{~mm}$, the resulting image, depicted in Fig. 4, shows a desaturating circle of $30 \mathrm{~mm}$ in diameter. The explanation for this phenomenon can be found in the material of the fluxgate core which

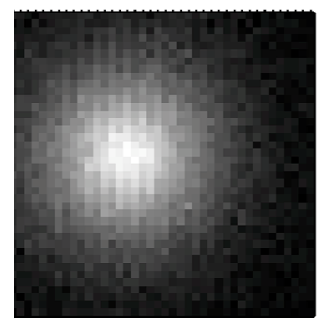

Figure 3: Scanning result of a sample of $8 \mathrm{~mm}$ in diameter. The imaged area is $40 \mathrm{~mm} \times 40 \mathrm{~mm}$.

consists of permalloy. This material has a very high magnetic permeability so that the magnetic field lines get attracted by the fluxgate core. This means that the fluxgate sensor also senses the magnetic field of a sample in some distance. Objects with sharp edges like the previous mentioned example are reproduced with a fading contrast.

(a)

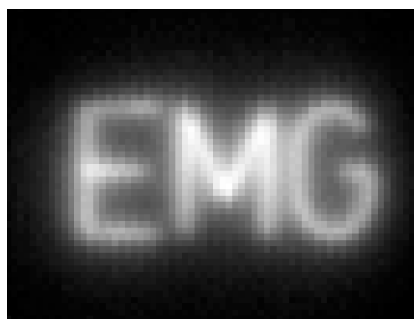

(b)

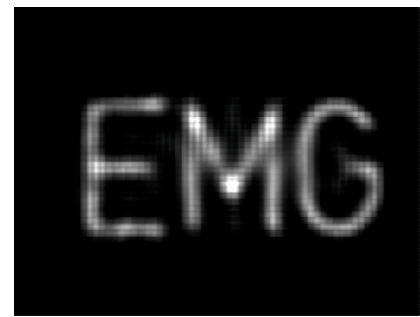

(c)

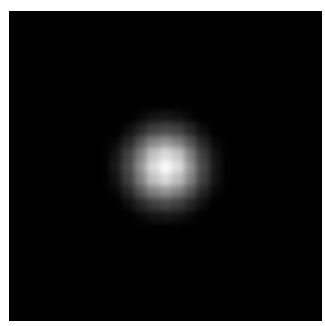

Figure 5: Sample image for deconvolution. (a) Raw scannng results, (b) deconvolved results, (c) measured PSF. 
Mathematically speaking, the resulting images are convolved with the aperture curve of the sensor. If, similarly to the approach to microscopy, the imaging result of a point-shaped sample - the so called pointspread function (PSF) - is known, the scanned image can be deconvolved and thus the resolution is significantly improved. We have designed a special sample to measure the point-spread function of our scanning device and evaluated the deconvolution using the microscopy software "ImageJ". The resolution of our images was greatly improved. In Fig. 5 an example is shown. The shown sample consists of freeze-dried and thereby immobilized nanoparticles which were positioned into engraved grooves forming our institute's logo "EMG". The size of this image is $6 \mathrm{~cm} \times 4 \mathrm{~cm}$ with a spatial resolution of $1 \mathrm{~mm}$. The spatial resolution of the magnetic nanoparticle distribution at the inner wall of the bag measured with this method now amounts to a few millimeters $(1-2 \mathrm{~mm})$ whereas the field resolution is of the order of 400 $\mathrm{pT}$, which is superior to other scanning methods like magnetic force microscopy.

\section{Optimization of system performance}

The system performance was evaluated in a test series with changing mechanical parameters. As expected, we have found that the vertical distance between sensor and sample surface is crucial. With a larger distance the spatial resolution deteriorates quickly as can be seen in Fig. 6.

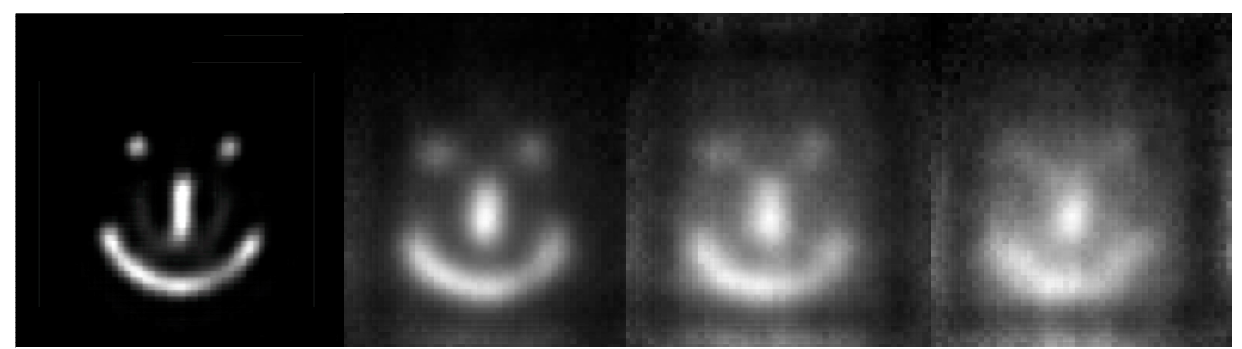

Figure 6: From left to right, scanning images for four different vertical distances between sensor and sample $(4 \mathrm{~cm} \times 4 \mathrm{~cm})$ are shown, starting at $0 \mathrm{~mm}$ and increasing by $1 \mathrm{~mm}$ from image to image. Spatial resolution deteriorates quickly as can be seen.

Nanoparticles bound to the modified cell culture bag surfaces were simulated by using overhead transparencies which were coated with nanoparticles. After having brushed the nanoparticles on the transparencies the samples were air-dried. Although - in contrast to experiments with real cell culture bags - there were not any unbound particles in the samples, they were still useful for analyzing the resolution and for determining the measurement threshold. A small patch of $1 \mu \mathrm{l}$ of a solution with a solid content of $10 \mathrm{mg} / \mathrm{ml}$ forming a circle of $2 \mathrm{~mm}$ in diameter could easily be measured.

(a)

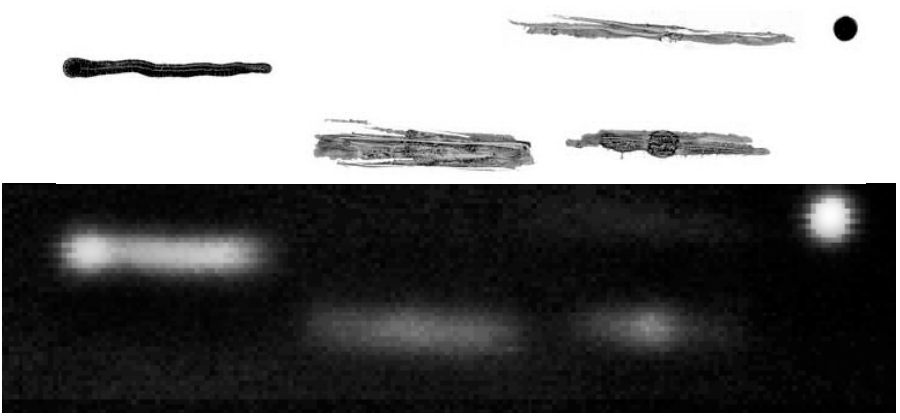

Figure 7: (a) Overhead transparancy $(2.5 \mathrm{~cm} \times 7 \mathrm{~cm})$ with brushed air-dried nanoparticles (top) and (b) MRX scan results (bottom).

Analysis of the images showed that the resolution - though already satisfactory - can be further improved: It seems that the used fluxgate magnetometer geometry is the limiting factor of resolution. 


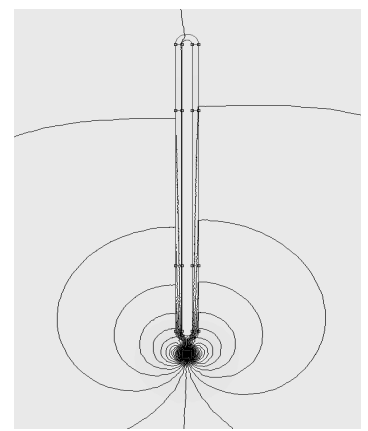

Figure 8: FEM-simulation of magnetic flux density in the fluxgate-core caused by a magnetic dipole with moment in z-direction.

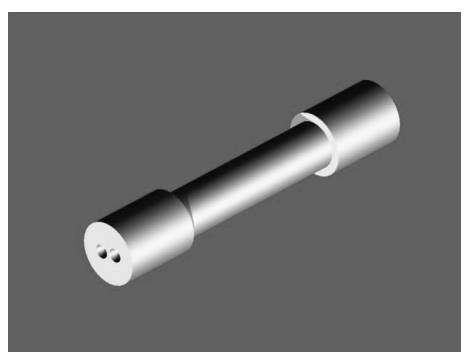

Figure 9: New winding form that guarantees fixed geometric positioning of the coils. Cylindrical excitation coils and core are placed inside the two parallel holes.

By simulating mechanical fluxgate properties we found out that the racetrack core has to be very narrow and that positioning the secondary coil at the bottom of the fluxgate close to the sample seems to be auspicious. The schematic setup is depicted in Fig. 1(b). As can be seen, different parameters have to be taken into consideration for optimizing the spatial resolution. While the length $I_{\text {prim }}$ and the number of turns $N_{\text {prim }}$ of the primary coils are not deciding, especially the length $I_{s e c}$ and the position of the secondary coil as well as the width $w$ and the number of turns $N_{\text {core }}$ of the core material are important. We studied these effects using the free software "FEMM". A typical example is depicted in Fig. 8. Obviously the fluxgate core is very inhomogeneously magnetized by the magnetic dipole.

Studying different coil positions inside the fluxgate requires them to be geometrically fixed. For that reason we designed a new winding form depicted in Fig. 9 that guarantees fixed mechanical coil positioning. It is made from PVC which has a low thermal capacity which might cause problems when large primary currents are used to magnetize the fluxgate core. Both the core and the coils could heat up quickly resulting in poor signal-to-noise-ratio. Noise measurements during the first minutes of fluxgate operation were performed showing that no thermal problems occur when moderate magnetizing currents of up to $80 \mathrm{~mA}$ are applied.

Measurements are in progress.

The work was financially supported by the German Ministry of Economy and Technology under Contract Number $16 \mathrm{INO548.}$

[1] F. Ludwig, S. Mäuselein, E. Heim, and M. Schilling, „Magnetorelaxometry of magnetic nanoparticles in magnetically unshielded environment utilizing a differential fluxgate arrangement", Rev. Sci. Instrum. 76, $106102(2005)$

[2] R. Piel, F. Ludwig, and M. Schilling, "Noise optimization of racetrack fluxgate sensors", Sensor Letters (submitted)

[3] E. Heim, S. Harling, K. Pöhlig, F. Ludwig, H. Menzel, and M. Schilling, „Fluxgate magnetorelaxometry of superparamagnetic nanoparticles for hydrogel characterization”, J. Magn. Magn. Mater. * $311^{\star}, 150-$ $154(2007)$ 